G. PITIS

KODAI MATH. J.

9 (1986), 327-333

\title{
ON SOME SUBMANIFOLDS OF A LOCALLY PRODUCT MANIFOLD
}

\author{
By GHeORghe Pitis
}

An investigation of properties of submanifolds of the almost product or locally product Riemannian manifolds has been started in the last years, many interesting results being obtained. So, Okumura [8], Adati and Miyazawa [1], Miyazawa [7], studied the hypersurfaces of such manifolds, Adati [2], defined and studied the invariant, anti-invariant and non-invariant submanifolds, while Bejancu [4], analyzed the semi-invariant submanifolds which are corresponding to CR-submanifolds of a Kaehler manifold [3].

The purpose of this paper is to give some properties of the anti-invariant and semi-invariant submanifolds, by using cohomology groups.

In $\S 1$ we recall the definition of these submanifolds and some known results, already. An example of semi-invariant submanifold is given.

In $\S 2$ we associate to a semi-invariant submanifold a de Rham cohomology class (as in [5] for CR-submanifolds) and we obtain a connection between the properties of the invariant and anti-invariant distributions and the cohomology of the submanifold (theorem 2.2).

The stability of some anti-invariant submanifolds of a locally product Riemannian manifold is studied in $\S 3$ and we give algebraic conditions for stability.

§1. Anti-invariant and semi-invariant submanifolds of a locally product Riemannian manifold. Let $(\tilde{M}, g, F)$ be a $C^{\infty}$-differentiable almost product Riemannian manifold, where $g$ is a Riemannian metric and $F$ is a non-trivial tensor field of type (1.1). Moreover $g$ and $F$ satisfy the following conditions

$$
F^{2}=I, \quad(F \neq \pm I) ; \quad g(F X, F Y)=g(X, Y), \quad X, Y \in \mathscr{X}(\tilde{M})
$$

where $I$ is the identity and $\mathscr{X}(\tilde{M})$ is the Lie algebra of vector fields on $\tilde{M}$.

We denote by $\tilde{\nabla}$ the Levi-Civita connection on $\tilde{M}$ with respect to $g$ and furthermore we assume that $\tilde{M}$ is locally product, that is

$$
\tilde{\nabla}_{X} F=0 \quad X \in \mathfrak{X}(\tilde{M}) .
$$

Let $M$ be a Riemannian manifold isometrically immersed in $\tilde{M}$ and denote

Received June 26, 1985 
by the same symbol $g$ the Riemannian metric induced on $M$.

If on $M$ there exist two complementary and orthogonals distributions $D$ and $D^{\perp}$, satisfying the following conditions:

$$
F\left(D_{x}\right)=D_{x} ; \quad F\left(D_{x}^{\perp}\right) \subset T_{x} M^{\perp} \quad \text { for each } \quad x \in M
$$

then $M$ is called a semi-invariant submanifold of the locally product Riemannian manifold $\tilde{M}$ [4].

Particularly if $\operatorname{dim} D_{x}=\operatorname{dim} T_{x} M\left(\operatorname{dim} D_{x}=0\right)$ for each $x \in M$ then $M$ is an invariant submanifold (anti-invariant submanifold) of $\tilde{M}$ [2].

We have the following results

THEOREM 1.1. [2] In a 2n-dimensional locally product Riemannian manifold every anti-invariant submanifold of dimension $n$ is totally geodesic.

THEOREM 1.2. [4] For a semi-invariant submanifold of a locally product Riemannan manifold the following assertions hold:

a) the distribution $D^{\perp}$ is involutive if and only if

$$
g(h(Y, Z), F X)=0 \quad X, Y \in D^{\perp}, \quad Z \in D
$$

where $h$ denote the second fundamental form of $M$;

b) $M$ is $D$-geodesic (i.e. $h(X, Y)=0$ for $X, Y \in D$ ) if and only if $D$ is involutive and each leaf of $D$ is totally geodesic immersed in $\tilde{M}$;

c) the distribution $D$ is involutive if and only if

$$
h(X, F Y)=h(F X, Y) \quad X, Y \in D \text {. }
$$

Example. Let $M$ be a normal metric almost paracontact manifold [9], [10], with the structure $(\varphi, \xi, \eta, g)$ and denote by $D^{*}$ the distribution $\{X \in T M: \eta(X)=0\}$. It is well-known that by putting

$$
F X=\varphi(X), \quad X \in D^{*} ; \quad F \xi=\frac{d}{d t} ; \quad F\left(\frac{d}{d t}\right)=\xi
$$

$F$ defines an almost product metric structure on $M \times R$. The product metric on $M \times R$ satisfies condition (1.1) and as $M$ is normal, it follows that $M \times R$ with this structure is local product. $M$ is a closed submanifold of $M \times R$ and from (1.3) follows that $F \xi \in T M^{\perp}, F D^{*}=D^{*}$ and then $M$ is semi-invariant in $M \times R$.

§ 2. Cohomology of semi-invariant submanifolds. Furthermore we assume $M$ as a compact without boundary manifold.

If $\operatorname{dim} D^{\perp}=q$ and $\operatorname{dim} D=p$ then we denote by $\mathscr{B}_{D^{\perp}}=\left\{X_{1}, \cdots X_{q}\right\}, \mathscr{B}_{D}=$ $\left\{X_{q+1}, \cdots, X_{q+p}\right\}$ two orthonormal local bases in $D^{\perp}$, resp. in $D$.

Proposition 2.1. If the distribution $D^{\perp}$ is involutive, then each leaf of $D^{\perp}$ is minimal in $M$. 
Proof. The mean-curvature vector of $D^{\perp}$ is

$$
H_{D^{\perp}}=\frac{1}{q} \sum_{\imath=1}^{q}\left(\nabla_{X_{i}} X_{\imath}\right)^{\perp}
$$

where $\left(\nabla_{X} X\right)^{\perp}$ is the component of $\nabla_{X} X$ in $D$.

By applying the Gauss formula

$$
\tilde{\nabla}_{X} Y=\nabla_{X} Y+h(X, Y) \quad X, Y \in \mathscr{X}(M)
$$

where $\nabla$ is the Riemannian connection on $M$, induced by $\tilde{\nabla}$, from the conditions (1.1) and (1.2) we have

$$
g\left(Y, \nabla_{X} X\right)=g\left(F Y, \tilde{\nabla}_{X}(F X)\right), \quad X \in \mathscr{B}_{D^{\perp}}, \quad Y \in D
$$

Now, by using the Weingarten formula

$$
\tilde{\nabla}_{X} N=-A_{N} X+\nabla_{X}^{\frac{1}{X}} N \quad N \in T M^{\perp}
$$

from (2.2) we can deduct

$$
g\left(Y, \nabla_{X} X\right)=-g\left(F Y, A_{F X} X\right)
$$

and taking into account the known equality

$$
g(h(X, Y), N)=g\left(A_{N} X, Y\right)
$$

we can write

$$
g\left(Y, \nabla_{X} X\right)=-g(h(X, F Y), F X)
$$

From (2.6) and the theorem 1.2 , a) follows $g\left(Y, \nabla_{X} X\right)=0$ and then $H_{D^{\perp}}=0$.

Q.E.D.

Proposition 2.2. For a D-geodesic semi-invariant submanifold the distribution $D$ is minimal.

Proof. If $X \in \mathscr{B}_{D}$ then there exists $\bar{X} \in D$ such that $F \bar{X}=X$ and from (1.1), (1.2), (2.1), (2.3), (2.5) we obtain

$$
g\left(Y, \nabla_{X} X\right)=g(F Y, h(F \bar{X}, \bar{X})), \quad Y \in D^{\perp} .
$$

But $M$ is $D$-geodesic and then from (2.7) follows $g\left(Y, \nabla_{X} X\right)=0$, hence $D$ is minimal.

Q.E. D.

We define on $M$ the 1 -forms $\omega^{1}, \cdots, \omega^{q}$, satisfying the following conditions

$$
\omega^{\imath}(Z)=0, \quad Z \in D ; \quad \omega^{\imath}\left(X_{j}\right)=\delta_{\jmath}^{\imath}, \quad X_{\jmath} \in \mathscr{B}_{D^{\perp}} ; \quad \imath, \jmath \in \overline{1, q} .
$$

Then we give the $q$-form $\omega=\omega^{1} \wedge \cdots \wedge \omega^{q}$, globally defined on $M$ and we have 
the following

Proposition 2.3. If the distribution $D$ is involutive and $D^{\perp}$ is minimal then the q-form $\omega$ is closed.

Proof. It is enough to prove that

$$
\begin{aligned}
& d \omega\left(Y, X_{1}, \cdots, X_{q}\right)=0 \quad \text { for } \quad Y \in D, \quad X_{1}, \cdots, X_{q} \in \mathscr{B}_{D^{\perp}} \\
& d \omega\left(Y_{1}, Y_{2}, X_{1}, \cdots, X_{q-1}\right)=0 \quad \text { for } \quad Y_{1}, Y_{2} \in D, \quad X_{1}, \cdots, X_{q-1} \in \mathscr{B}_{D^{\perp}} .
\end{aligned}
$$

From the definition of the forms $\omega^{2}$ it follows

$$
d \omega\left(Y, X_{1}, \cdots, X_{q}\right)=\sum_{\imath=1}^{q} g\left(\left[Y, X_{\imath}\right], X_{\imath}\right)
$$

Now, the connection $\tilde{\nabla}$ is Riemannian and then

$$
g\left(X_{\imath}, \tilde{\nabla}_{Y} X_{\imath}\right)=0 \quad X_{i} \in \mathscr{B}_{D^{\perp}}, \quad Y \in D .
$$

Taking into account (2.12), (2.1) follows

$$
g\left(\left[Y, X_{\imath}\right], X_{\imath}\right)=g\left(\nabla_{X_{i}} X_{\imath}, Y\right)
$$

But $D^{\perp}$ is minimal and then from (2.11) and (2.13) we obtain (2.9).

The distribution $D$ being involutive, (2.10) follows from the equality

$$
d \omega\left(Y_{1}, Y_{2}, X_{1}, \cdots, X_{q-1}\right)=-\omega\left(\left[Y_{1}, Y_{2}\right], X_{1}, \cdots, X_{q-1}\right) .
$$
by

In the same manner as above we can define on $M$ the 1 -forms $\theta^{q+1}, \cdots, \theta^{q+p}$

$$
\theta^{q+\imath}(Z)=0, \quad Z \in D^{\perp} ; \quad \theta^{q+\imath}\left(X_{q+j}\right)=\delta_{j}^{\imath}, \quad X_{q+j} \in \mathscr{B}_{D}, \quad i, j \in \overline{1, p} .
$$

By using a similar computation as in the proof of the proposition 2.3 we can state the following

Proposition 2.4. If $D$ is minimal and if $D^{\perp}$ is involutive then the p-form $\theta=\theta^{q+1} \wedge \cdots \wedge \theta^{q+p}$ is closed on $M$.

Now from Propositions $2.3,2.4$ we have $\theta=* \omega$ and by applying the Hodgede Rham theorem we obtain the

THEOREM 2.1. For any compact semi-invarıant submanifold $M$, of a Riemannian locally product manifold, having the distribution $D$ involutive and $D^{\perp}$ minimal, a cohomology de Rham class $[\omega] \in H^{q}(M, R)$ is well-defined. This class is non trivial if $D$ is minimal and $D^{\perp}$ is involutive.

From Proposition 2.1 and Theorem 2.1 follows 
ON SOME SUBMANIFOLDS OF A LOCALLY PRODUCT MANIFOLD

THEOREM 2.2. Let $M$ be a compact semi-invariant submanifold of a locally product Riemannian manifold. If the distributions $D, D^{\perp}$ are involutıves and $D$ is minimal then

$$
H^{q}(M, R) \neq 0 \quad q=\operatorname{dim} D^{\perp} .
$$

From Theorems 1.2, 2.2 and Proposition 2.2 we deduce the following

Proposition 2.5. For every compact and totally geodesic semi-invariant submanifold of a locally product Riemannian manifold the Bettr number $b_{q}, q=$ $\operatorname{dim} D^{\perp}$, not vanish.

Now taking into account the Theorem 2.2 and the above example we obtain the following

PROPOSITION 2.6. Let $M$ be a compact normal metric almost paracontact manifold. If the distribution $D^{*}$ is involutive and minimal then

$$
H^{1}(M, R) \neq 0
$$

Remark. It is well-known that if $M$ is a SP-Sasakian manifold then the distribution $D^{*}$ is involutive [9], [10].

Next we can make some comments on the obtained results.

A. Let $S^{2 n+1}$ be the unit sphere in $R^{2 n+2}, n \geqq 2$, endowed with the standard Sasakian structure $(f, \xi, \eta)$. It is known that the tensor field $F$ given by

$$
g(F X, Y)=\left(\nabla_{X} \eta\right) Y
$$

defines a SP-Sasakian structure on $S^{2 n+1}$. Moreover $H^{1}\left(S^{2 n+1}, R\right)=0$ for $n \geqq 1$ and then the sphere $S^{2 n+1}, n \geqq 2$, is a semi-invariant submanifold of $S^{2 n+1} \times R$, so that the distribution $D^{*}$ is not minimal.

B. Suppose $M$ is a compact SP-Sasakian manifold totally geodesic immersed in the Riemannian locally product manifold $M \times R$. From Theorem 1.2 and Proposition 2.2 it follows that the distribution $D^{*}$ is involutive and minimal. Hence the first Betti number of $M$ not vanish.

§ 3. Stability of anti-invariant submanifolds. Let $M$ be a compact $n$ dimensional anti-invariant submanifold of the $2 n$-dimensional locally product Riemannian manifold $\tilde{M}$.

By applying (2.1), (2.3) and Theorem 1.1 we have the

LEMma 3.1. For every $X, Y \in T M$ the next equalities holds

$$
\nabla_{\bar{Y}}^{\frac{1}{}}(F X)=F\left(\nabla_{Y} X\right) \quad A_{F X} Y=0 .
$$

Let $\left\{X_{1}, \cdots, X_{n}\right\}$ be a orthonormal local basis in $T M$ and lets denote by $S$ and $\widetilde{S}$ the Ricci tensors associated to the manifolds $M$ and $\tilde{M}$. 
LEMMA 3.2. For every $X \in T M$ we have

$$
\sum_{\imath=1}^{n} \tilde{R}\left(X_{\imath}, F X, F X, X_{\imath}\right)=\tilde{S}(X, X)-S(X, X) .
$$

Proof. From (1.1), (1.2) it follows that

$$
\tilde{R}\left(X_{\imath}, F X, F X, X_{\imath}\right)=\tilde{R}\left(F X_{\imath}, X, X, F X_{\imath}\right) .
$$

Now the required equality is a consequence of (3.1) and of the Gauss equation

$$
\begin{aligned}
R(U, V, W, T)= & \tilde{R}(U, V, W, T)+g(h(U, T), h(V, W)) \\
& -g(h(U, W), h(V, T)) .
\end{aligned}
$$

Let $N$ be a normal vector field and denote by $C V^{\prime \prime}(N)$ the second normal variation of $M$ induced by $N$. Then we have ([6], chap. I)

$$
c V^{\prime \prime}(N)=\int_{M}\left\{\left\|\nabla^{\perp} N\right\|^{2}-\sum_{\imath=1}^{n} \tilde{R}\left(X_{\imath}, N, N, X_{\imath}\right)-\left\|A_{N}\right\|^{2}\right\} d V
$$

where $d V$ is the volume form of $M$.

On the other hand if $\eta$ is the 1 -form associated to the vector field $X \in T M$ then we have the well-known formula ([6], chap. V)

$$
\int_{M}\left\{S(X, X)+\|\nabla X\|^{2}-\frac{1}{2}\|d \eta\|^{2}-(\delta \eta)^{2}\right\} d V=0 .
$$

Taking into account the Lemma 3.1 and the Theorem 1.1, from (3.3), (3.4) follow the

PROPOSITION 3.1. The normal variation induced by the normal vector field $N=F X$ of the compact anti-invariant submanifold $M$ in a locally product Riemannian manifold is given by

$$
\mathcal{V}^{\prime \prime}(N)=\int_{M}\left\{\frac{1}{2}\|d \eta\|^{2}+(\delta \eta)^{2}-\tilde{S}(N, N)\right\} d V .
$$

Now we can state the following

THEOREM 3.1. Let $M$ be a compact anti-invariant submanfold of the locally product Riemannian manifold $\tilde{M}$.

a) If $\widetilde{S}$ is negative definite then $M$ is stable.

b) If $H^{1}(M, R) \neq 0$ and $\widetilde{S}$ is positive definite then $M$ is unstable.

Proof. a) is an immediate consequence of the Proposition 3.1 because we obtain $C \mathcal{V}^{\prime \prime}(N)>0$ for every $N \in T M^{\perp}$.

b) Since $H^{1}(M, R) \neq 0$ there exists an harmonic 1 -form $\eta$ on $M$ and if $X$ is it associated vector field, we have $d \eta=\delta \eta=0$ and then $\mathcal{C V}^{\prime \prime}(F X)<0$. Consequently $M$ is unstable in $\tilde{M}$. 


\section{REFERENCES}

[1] T. Adati and T. Miyazawa, Hypersurfaces immersed in an almost product Riemannian manifold, TRU Math., 14-2 (1978), 17-26.

[2] T. AdATI, Submanifolds of an almost product Riemannian manifold, Kodai Math. J., 4-2 (1981), 327-343.

[3] A. Bejancu, CR-submanifolds of a Kaehler manifold I, Proc. Amer. Math. Soc., 69 (1978), 135-142.

[4] A. BEJANCu, Semi-invariant submanifolds of locally product Riemannian manifolds, Ann. Univ. Timisoara, s. Math., XXII (1984), 3-11.

[5] B. Y. CHEN, Cohomology of CR-submanifolds, Ann. Fac. Sci. Toulouse, 3 (1981), $167-172$.

[6] B. Y. Chen, Geometry of submanifolds and its applications, Science University of Tokyo, 1981.

[7] T. MiYazawa, Hypersurfaces immersed in an almost product Riemannian manifold, Tensor N.S., 33-1 (1979), 114-116.

[8] M. OKumuRa, Totally umbilical hypersurfaces of a locally product Riemannian manifold, Kodai Math. Sem. Rep., 19 (1967), 35-42.

[9] I. SATo, On a structure similar to the almost contact structure, Tensor N.S. 30 (1976), 219-224; 31 (1977), 199-205.

[10] I. SATo, On a Riemannian manifold admitting a certain vector field, Kodai Math. Sem. Rep., 29 (1978), 250-260.

UNIVERSITY OF BRASOV

Faculty of Mathematics

2200 Brasov, Romania 